\title{
Macrophage activation syndrome (MAS) in different pediatric rheumatic disease
}

\author{
Zubeyde Gunduz', Betul Sozeri ${ }^{1,2^{*}}$, Ruhan Dusunsel', Hakan Poyrazoglu', Ismail Dursun', Aysenur Pac Kisaarslan', \\ Sibel Yel ${ }^{1}$, Kenan Yilmaz ${ }^{1}$ \\ From 21st European Pediatric Rheumatology (PReS) Congress \\ Belgrade, Serbia. 17-21 September 2014
}

\section{Introduction}

Macrophage activation syndrome (MAS) is a life-threatening complication of chronic rheumatic disease in childhood.

\section{Objectives}

We aim to eveluate MAS findings and outcomes that differ according to disease in childhood.

\section{Methods}

We obtain 11 rheumatic patients followed in two different pediatric rheumatology units (Erciyes University and Ege University) who presented with MAS. We report their clinical and laboratory findings, therapies and outcomes.

\section{Results}

The primary diagnoses of the patients included in the study, respectively; systemic juvenil idiopathic arthritis $(\mathrm{n}=5)$, Systemic Lupus Erythematosus $(\mathrm{n}=2)$, juvenile dermatomyositis $(n=2)$, a neonatale onset multisystem inflammatory disease (NOMID) and a microscopic polyartritis nodosa. The mean age of the patients was 9.9 years old (1-14), and male to female ratio was $3: 8$. The mean duration of underlying disease was 6 months (1-24 months) at the diagnosis of MAS. We found MAS due to infection in four patients, while used medicine in a patient. MAS were developed spontaneously in 6 patients. The clinical manifestations of MAS included fever 7 (63.6\%),mucosal bleading 6 (54.5\%), neurologic involvement 4 (36.4\%) and hepatomegaly 6 (54.5\%).

We found thrombocytopenia in $9(81.8 \%)$, leucopenia in $5(45.5 \%)$, increased AST in 7 (63.6\%), hypofibrinogenemia in 6 (54.5\%), increased ferritin in $11(100 \%)$, decreased ESH in $4(36.4 \%)$ and increased triglyceride in $10(90.9 \%)$ patients. We investigated bone marrow in all patients, and hemophagocytosis were determinated in $8(72.7 \%)$. The medications were pulse methylprednisolone 6 (54.5\%), intravenous immunoglobulin $8(72.7 \%)$, plasma exchange $5(45.5 \%)$, cyclosporine 6 (54.5\%), dexamethasone 1 (9.1\%), etoposide $1(9.1 \%)$. The prognosis of patients were recovery $8(72.7 \%)$, and exitus 3 (27.3\%).

\section{Conclusion}

In conclusion, MAS can be developed in various pediatric rheumatologic disease and fatal. Prompt recognition and timely treatment can result good outcomes.

\section{Disclosure of interest}

None declared.

\section{Authors' details}

${ }^{1}$ Pediatric Rheumatology, Erciyes University, Kayseri, Turkey. ${ }^{2}$ Pediatric Rheumatology, Ege University, Izmir, Turkey.

Published: 17 September 2014

doi:10.1186/1546-0096-12-S1-P220

Cite this article as: Gunduz et al.: Macrophage activation syndrome (MAS) in different pediatric rheumatic disease. Pediatric Rheumatology 2014 12(Suppl 1):P220.

'Pediatric Rheumatology, Erciyes University, Kayseri, Turkey

Full list of author information is available at the end of the article 